\title{
A Randomised Controlled Trial (RCT) evidence of a telehealth approach for suicide prevention in Sri Lanka: an intervention study
}

\author{
Dr. R.B. Marasinghe \\ Department of Medical Education, University of Sri Jayewardenepura, Sri Lanka.
}

\section{Dr. S. Edirippulige}

Centre for Online Health, University of Queensland, Australia.

\author{
Dr. A. Smith \\ Centre for Online Health, University of Queensland, Australia.
}

Dr. D. Kavanagh

Institute of Health \& Biomedical Innovation, Queensland University of Technology, Australia.

Prof. M.T.M. Jiffry

Department of Medical Education, University of Sri Jayewardenepura, Sri Lanka.

Contact e-mail address: rohanabm@yahoo.com

eHealth Sri Lanka 2010,1(suppl.1):S11

DOI: http://dx.doi.org/10.4038/sljbmi.v1i0.3544

Only the Abstract is available

\begin{abstract}
Introduction: Suicide rate in Sri Lanka is double the world's average but the healthcare resources remain insufficient. Tele-health and the delivery of healthcare over distance are being increasingly used globally. However, clinical effectiveness is yet to be established.

Objective: Main objective of this study was to test whether a Brief Mobile Treatment (BMT) can improve the outcome to a significant extent than Usual Care (UC) alone, among those who attempt suicide. Method: The BMT included a brief face-to-face training followed up by a mobile phone communication. The UC included whatever the care people have access to, in Sri Lanka. A total of 68 participants were randomly recruited from Colombo South Teaching Hospital in 2008. The results were measured at Baseline, 6 and 12 months, suicidal tendency, depression, social support and alcohol/drug use; using standardised instruments.

Results: Both groups did not have a significant variation at the Baseline $(\mathrm{p}>0.05)$. The results (i.e. suicidal tendency, depression, social support and substance use) were generally improved with time. With regard to suicidal tendency, depression and social support, Condition x Time was significant but neither Gender x Time nor Condition x Gender x Time were significant. With regard to substance use, there was a significant Gender x Time effect but no significant effect on either Condition $\mathrm{x}$ Time or Condition x Gender x Time. A differential effect was noted when the time was broken down into two non-orthogonal contrasts. With regard to suicidal tendency, depression and social support, the Condition $\mathrm{x}$ Time was significant over Baseline vs. 6 months but not significant over Baseline vs. 12 months but for alcohol/other drug use it was vary.

Conclusion: The Brief Mobile Intervention (BMT) was superior in reducing suicidal ideation and depression than the Usual Care (UC) but not effective in reducing actual self-harm and substance use. However, BMT is effective in improving social support.
\end{abstract}

Keywords - Suicide rate, Depression, social support, BMT 
